\title{
In Situ TEM for Electrochemical Energy Storage and Conversion Systems
}

\author{
Megan E. Holtz ${ }^{1}$, Yingchao Yu², Johary Rivera ${ }^{2}$, Héctor D. Abruña ${ }^{2}$, and David A. Muller ${ }^{1,3}$ \\ 1. School of Applied and Engineering Physics, Cornell University, Ithaca NY 14853. \\ 2. Department of Chemistry and Chemical Biology, Cornell University, Ithaca NY 14853. \\ 3. Kavli Institute at Cornell for Nanoscale Science, Ithaca, NY, USA
}

Advances in the lifetime and performance of batteries and fuel cells are required before they are cost effective for electric vehicles or as a way to level the supply of power from intermittent, renewable energy sources. Because both the materials and processes in these energy storage and conversion systems are inhomogeneous on the nanoscale, in situ (scanning) transmission electron microscopy (STEM) is well suited to provide dynamic, nanometer scale information [1]. Here we discuss platinum catalysts on carbon supports for fuel cell applications, observing the carbon flex during cycling. Finally, we present work observing the lithiation of a battery cathode material using in situ spectroscopy. We find that the mechanism of lithiation is different for different particles in the same aggregate.

The morphology of the support structure for catalysts in fuel cells is important in determining their degradation and transport behavior. We observe morphological changes in catalyst supports at varying potentials in a Protochips electrochemical cell in a $200 \mathrm{keV}$ Tecnai F20. Figure 1 shows platinum nanoparticles on high surface area carbon (HSC) in $0.1 \mathrm{M} \mathrm{H}_{2} \mathrm{SO}_{4}$. During cyclic voltammetry, the carbon aggregates swell at low potential and relax at high potential (Fig 1a-c). The carbon branch that is highlighted reversibly oscillates tens of nanometers with the voltage (Fig 1d). At low potential, the branch moves away from its neighbor, and then relaxes at high potential. We suspect that the dynamics of the HSC are of capacitive origin, attracting and possibly intercalating protons at negative potentials. The lower mobility and larger size of the sulfate anions inhibits similar behavior at high potentials. If the carbon oscillation occurs in a fuel cell, it may degrade gas diffusion pathways or lead to Pt coalescence. In a separate experiment, we observed carbon support degradation at high potentials leading to platinum coalescence. This suggests carbon support movement may adversely affect cell lifetimes.

In situ cyclic voltammetry of Pt nanoparticles on HSC is shown in Fig 1e. The working electrode, which contains the electrochemically active sample, is confined to the viewing membrane. Even with our small electrode and current scale ( $5 \mathrm{nA})$, the measurement has minimal noise. As the sweep rate is increased, the current increases roughly as the square root of the sweep rate. From hydrogen adsorption/desorption (HAD) measurements, we find roughly $2 \times 10^{-5} \mathrm{~cm}^{2}$ of platinum surface area on the electrode.

The study of the lithiation and delithiation of battery materials is critical because this mechanism often limits electrochemical performance [2]. Here, we use valence energy-filtered TEM (EFTEM) imaging of an aqueous battery cathode to track ions as they move between electrolyte and cathode material [1]. We find in the valence EELS signal that there is a peak at $5 \mathrm{eV}$ in $\mathrm{FePO}_{4}$ but not $\mathrm{LiFePO}_{4}(\mathrm{Fig} 2 \mathrm{a})$. From this, we can track the state of lithiation by imaging regions of the material that have lost $5 \mathrm{eV}$ in EFTEM mode. The discharged material, $\mathrm{LiFePO}_{4}$, appears dark in the $5 \mathrm{eV}$ image (Fig 2b,h). During charging (Fig $2 \mathrm{c}-\mathrm{g}$ ), regions of the particle light up, indicated delithiated $\mathrm{FePO}_{4}$. The bright regions disappear during discharging. We find two different mechanisms for delithiation, indicated by arrows in Fig 2 . We expect valence EFTEM will be effective for other energy related materials, since they undergo electronic structure changes of a few eV during operation. [3] 
References:

[1] M Holtz et al, NanoLetters 14 (2014) p. 1453.

[2] C.M. Wang, et al. Journal of Materials Research 25 (2010) p. 1541.

[3] Work supported by the Energy Materials Center at Cornell, DOE EFRC BES (DE-SC0001086). EM

Facility support from the NSF MRSEC program (DMR 1120296).
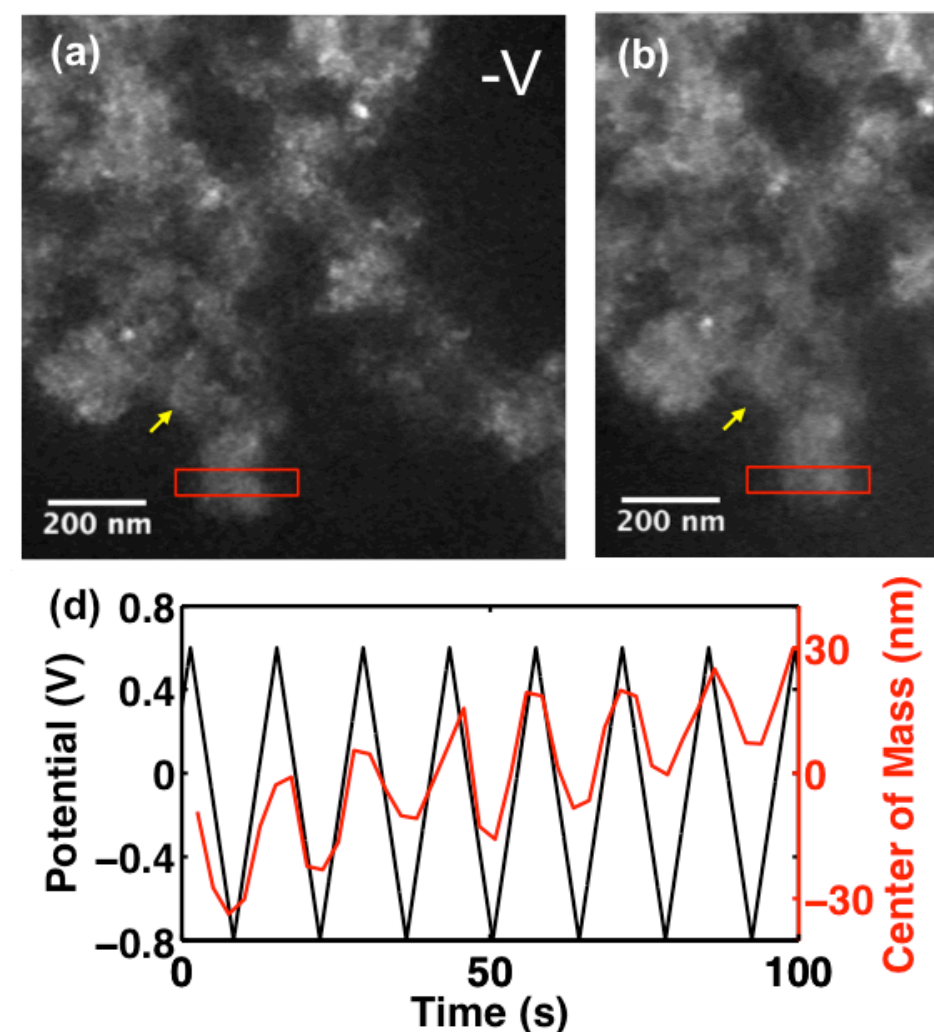
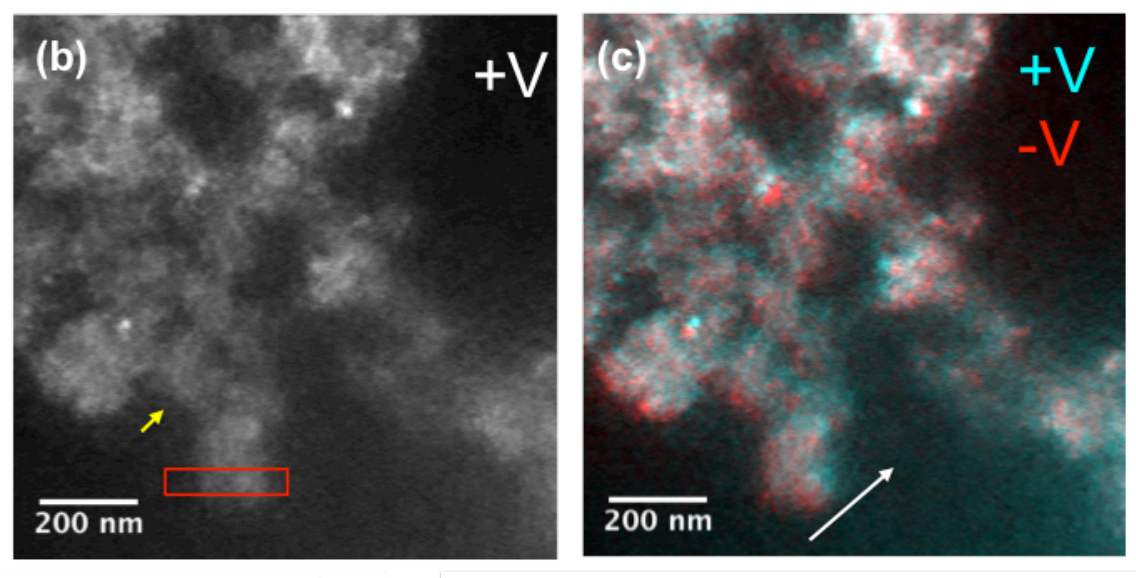

(e)

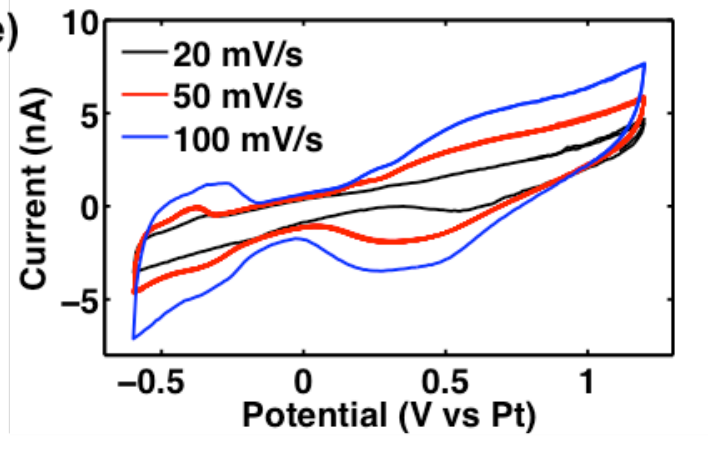

Figure 1. In situ cycling of Pt nanoparticles on HSC in $0.1 \mathrm{M} \mathrm{H}_{2} \mathrm{SO}_{4}$. HAADF-STEM images at (a) negative potentials and (b) positive potentials, with a color overlay in (c). At low potential, the carbon aggregates swell, suggesting proton intercalation, which relaxes at positive potentials. (d) Position of the particle marked by the red box, plotted with voltage. Particle moves more to the right at high potential. (e) Cyclic voltammetry of Pt with sweep rate, at low current density. HAD estimates $\sim 25 \mathrm{pg}$ of Pt.
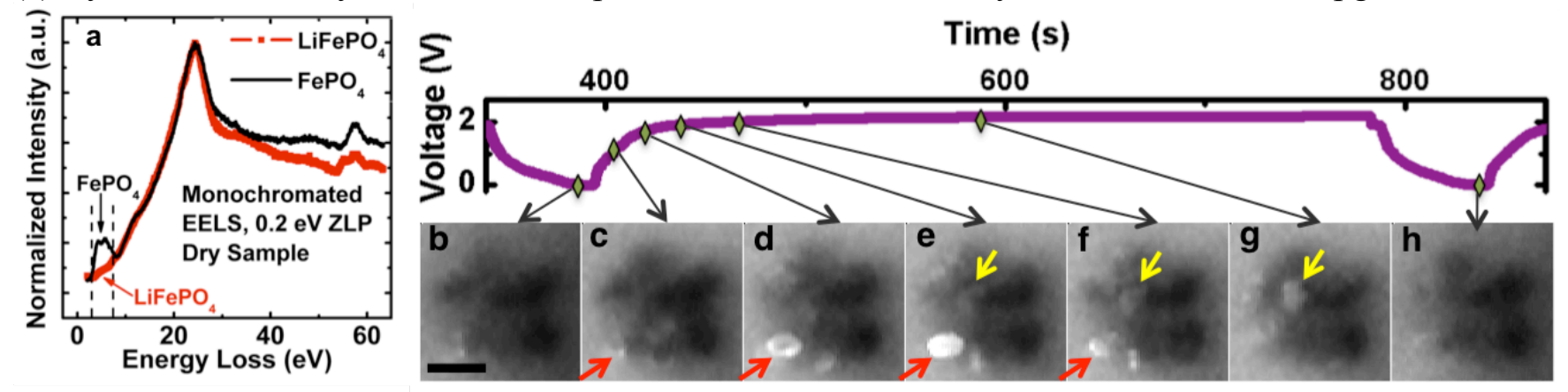

Figure 2. (a) EELS of $\mathrm{LiFePO}_{4}$ and $\mathrm{FePO}_{4}$ shows a peak at $5 \mathrm{eV}$ for $\mathrm{FePO}_{4}$. (b-h) Observation of the evolution of a cluster of $\mathrm{LiFePO}_{4} / \mathrm{FePO}_{4}$ during one charge/discharge cycle. EFTEM images formed at 5 $\mathrm{eV}$, highlighting the delithiated $\mathrm{FePO}_{4}$, during charge and discharge (voltage curve above). ( $\mathrm{b}$ and $\mathrm{h}$ ) are discharged $\mathrm{LiFePO}_{4}$. The red arrows in (c-f) correspond to core-shell structures, while yellow arrows (eg) show delithiation propagating in one direction through the particle. $200 \mathrm{~nm}$ scale bar. 